\title{
Efficacy and safety of stenting for elderly patients with severe and symptomatic carotid artery stenosis: a critical meta-analysis of randomized controlled trials
}

\section{Yi-An Ouyang \\ Yugang Jiang \\ Mengqiang Yu \\ Yunze Zhang \\ Hao Huang}

Department of Neurosurgery, Second Xiang-Ya Hospital of Central South University, Changsha, People's Republic of China
Correspondence: Yugang Jiang Department of Neurosurgery, Second Xiang-Ya Hospital of Central South University, No 139, Renmin Road, Changsha 4I00II, Hunan Province, People's Republic of China

Tel +8673185295888

Fax +8673185295888

Email ygjiangmd@I63.com
This article was published in the following Dove Press journal:

Clinical Interventions in Aging

28 October 2015

Number of times this article has been viewed

Objective: To investigate both short-term and long-term therapeutic efficacy and safety of carotid artery stenting (CAS) and carotid artery endarterectomy (CEA) for elderly patients with severe and symptomatic carotid artery stenosis.

Methods: PubMed, EMBASE, Cochrane Library, Clinical Trials Register Centers, and Google Scholar were comprehensively searched. After identifying relevant randomized controlled trials, methodological quality was assessed by using Cochrane tools of bias assessment. Meta-analysis was performed by RevMan software, and subgroup analyses according to different follow-up periods were also conducted.

Results: Sixteen articles of nine randomized controlled trials containing 6,984 patients were included. Compared with CEA, CAS was associated with high risks of stroke during periprocedural 30 days (risk ratio $[\mathrm{RR}]=1.47,95 \%$ confidence interval $[\mathrm{CI}]: 1.15-1.88$ ), 48 months ( $R R=1.37,95 \%$ CI: 1.11-1.70), and $>48$ months ( $R R=1.76,95 \%$ CI: 1.34-2.31). There was no significant difference in the aspects of death, disabling stroke, or death at any time between the groups. For other periprocedural complications, CAS decreased the risk of myocardial infarction ( $R R=0.44,95 \%$ CI: 0.26-0.75), cranial nerve palsy ( $R R=0.09,95 \%$ CI: $0.04-0.22)$ and hematoma $(\mathrm{RR}=0.31,95 \% \mathrm{CI}: 0.14-0.68)$ compared with $\mathrm{CEA}$, while it increased the risk of bradycardia or hypotension ( $\mathrm{RR}=8.45,95 \% \mathrm{CI} 2.91-24.58$ ).

Conclusion: Compared with CEA, CAS reduced hematoma, periprocedural myocardial infarction, and cranial nerve palsy, while it was associated with higher risks of both short-term and long-term nondisabling stroke. And they seemed to be equivalent in other outcome measures. As regards to its minimal invasion, it should be applied only in specific patients.

Keywords: symptomatic carotid artery stenosis, carotid artery stenting, carotid artery endarterectomy

\section{Introduction}

According to the latest statistic from the American Heart Association, stroke ranks third among all the death causes, and every 4 minutes someone dies of stroke. Of all the strokes, $87 \%$ are ischemic, and people from 55 to 75 years of age who have a risk of stroke is $14 \%$ for men and $20 \%$ for women in the USA. ${ }^{1}$

Carotid artery stenosis and occlusive diseases induced by many factors are important causes of ischemic stroke, and they often lead to immediate death although they count approximately $10 \%-15 \%$ of all the strokes. ${ }^{2}$ Symptomatic patients with a $>50 \%$ stenosis of vessel lumen was considered to be of high risk, and need to adopt aggressive treatments. ${ }^{3}$ Among the kinds of methods, carotid artery endarterectomy (CEA) was established as an effective option that periprocedural stroke/death 
is $<6 \%$ for symptomatic and $<3 \%$ for asymptomatic patients, and 10 -year risk of stroke after CEA is approximately $2 \%$ per year. ${ }^{4,5}$ Carotid artery stenting (CAS) that emerged in the past 2 decades has also gradually developed to be an important and minimally invasive alternative. Compared with CEA, it was supposed to enhance recovery, reduce complication, and achieve cosmetic effect, ${ }^{3}$ and CAS was performed increasingly in clinical practice during the past few years.

However, therapeutic efficacy and safety of CAS compared with CEA were still uncertain. Up-to-date, a series of randomized controlled trials (RCTs) were designed and performed with various participants and follow-up periods. ${ }^{6-21}$ As insufficient statistical test power existed in single trials, the results and conclusions across the trials were controversial and confusing. Meanwhile, due to the lack of long-term results of follow-up longer than 2 years, current meta-analyses based on periprocedural and short-term data $^{22,23}$ were not enough to provide valid and comprehensive evidence. Recently, in 2014 and 2015, many large-scale and multicenter RCTs stratified and published their long-term results ranged from postoperative $2-10$ years. ${ }^{11,17,18}$

It was necessary to take them together, and all the relevant RCTs involving short-term and long-term results could enhance our current knowledge and findings. So we conducted this critical and updated meta-analysis to conclude the comparative outcomes of CAS and CEA for carotid artery stenosis treatment.

\section{Methods}

\section{Literature search}

A comprehensive search was performed on the databases including PubMed (1966.01-2015.05), EMBASE (1974.012015.05), and Cochrane Library (2015 Issue 5), as well as Clinical Trials Register Centers (up to 2015.05). Search terms were as follows: ("carotid artery" OR "vertebrobasilar" OR "cerebral" OR "craniocerebral" OR "head and neck") AND ("angiostenosis" OR "stenosis" OR "obstruct” OR "endothelial thicken" OR "occlusive disease") AND ("stent" OR “stenting”). Medical subject headings were also used. Related articles, the references of relevant trials, and reviews were also screened to identify potential publications. Google Scholar was also searched for the lasted published articles.

\section{Inclusion and exclusion criteria}

Literature search results were first imported to citation manager software, and after duplication removed titles and abstracts were carefully scanned. At last, potential publications were further assessed by reading full-texts.
Publications were included if 1) RCTs investigated the therapeutic efficacy and safety of CAS and CEA in carotid artery stenosis; 2) symptomatic patients $>60$ years, and with a severe carotid artery stenosis $>50 \%$ of the luminal diameter, or asymptomatic patients with a $>60 \%$ stenosis were participants; 3 ) preoperative aspirin was begun at least 72 hours before CAS or CEA and was continued indefinitely in both groups. Standard CEA was performed, and the stent used was self-expanding-nitinol stent with an emboli-protection device; and 4) primary outcomes should at least include stroke, death, or both of them. Secondary outcomes should include other complications such as transient ischemic attack, cranial nerve palsy, hematoma, restenosis, infection, and artery thrombosis. Meeting abstracts, reviews, non-RCTs, and non-English published papers were excluded.

\section{Data collection and quality assessment}

Reviewers extracted baseline characters of the included trials, which contained the first author, published year, case, average age, interventions, stenosis severity, diagnosis determination methods, and follow-up period. Data of outcomes were extracted in a predesigned table for pooled analysis. Methodological quality was assessed by the tool of bias assessment provided by Cochrane Collaboration, which was based on six items: ${ }^{23}$ randomization, allocation concealment, participant, outcome assessment blinding, incomplete outcomes, selective reporting, and other bias. All data extraction and quality assessment were performed by two reviewers independently. Any disagreements were resolved by a third reviewer.

\section{Statistical analysis}

Meta-analysis was performed by using RevMan software (version 5.3, the Cochrane Collaboration, Copenhagen, Denmark). Subgroup analyzes were performed to identify important clinical characters, and all the analyzes were first performed based on clinical homogeneity. After that, $\chi^{2}$ and $I^{2}$ statistical tests were used to judge and present the statistical heterogeneity across the trials. A homogeneity was considered when $I^{2} \leq 50 \%$, and fixed-effects model was chosen. Random-effects model was chosen when a heterogeneity existed, $I^{2}>50 \%$. Risk ratio (RR) and mean difference with their respective 95\% confidence intervals (CIs) were presented for pooled effect size. Invested funnel plots were used to assess the risks of publication bias.

The meta-analysis was reported mainly according to Preferred Reporting Items for Systematic Reviews and 
Meta-Analyses: the PRISMA statement. ${ }^{24}$ It did not involve any ethic issues.

\section{Results}

\section{Trial inclusion and quality assessment results}

Finally, 16 articles $^{6-21}$ of nine trials containing 6,984 patients were included. There were 3,511 cases in the CAS group and 3,473 cases in the CEA group. Flow diagram of trial selection from initial search result to final decision is shown in Figure 1. The baseline characteristic of the included trials was presented in Table 1. Except three trials included both symptomatic and few asymptomatic patients, ${ }^{6,7,14,19,20}$ the others only included symptomatic patients. The three trials included patients who suffered $>50 \%$ internal carotid artery (ICA) stenosis, ${ }^{6,7,18-20}$ and the four trials included patients who suffered $>70 \%$ ICA stenosis. ${ }^{8,9,13,15-17}$ Follow-up ranged from postprocedural 30 days to 10 years.

Methodological quality assessment result was shown in Figure 2 . The overall quality was good, whereas the item of blinding of participants and personnel was under unclear risk of bias. As a comparison of surgery, the procedure of CAS and CEA was really different, and blinding of participants and personnel was hard to realize.

\section{Primary periprocedural and follow-up results \\ Death}

According to the different follow-up period, a subgroup analysis including periprocedural 30 days, postprocedural 24,48 , and $>48$ months was conducted. Meta-analysis results

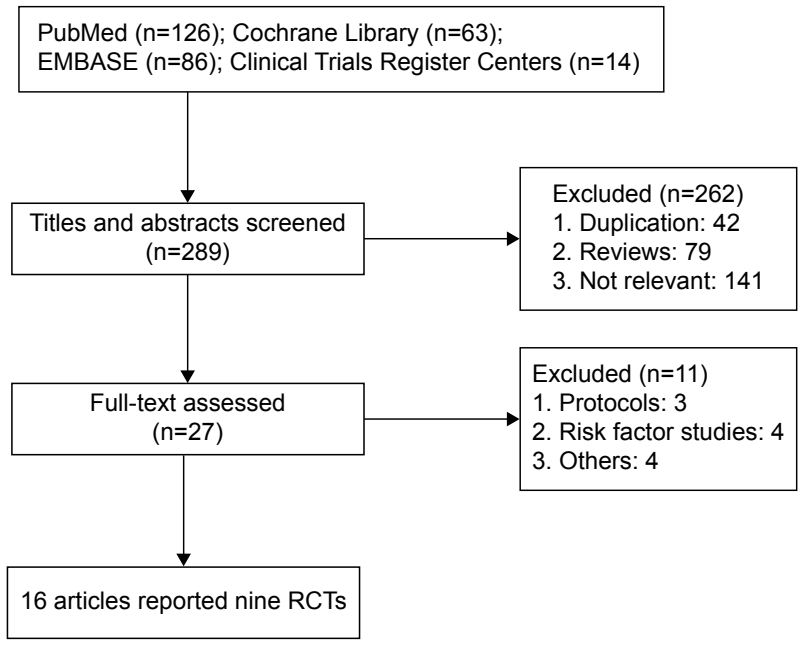

Figure I Flow diagram of trials selection. Abbreviation: RCTs, randomized controlled trials.

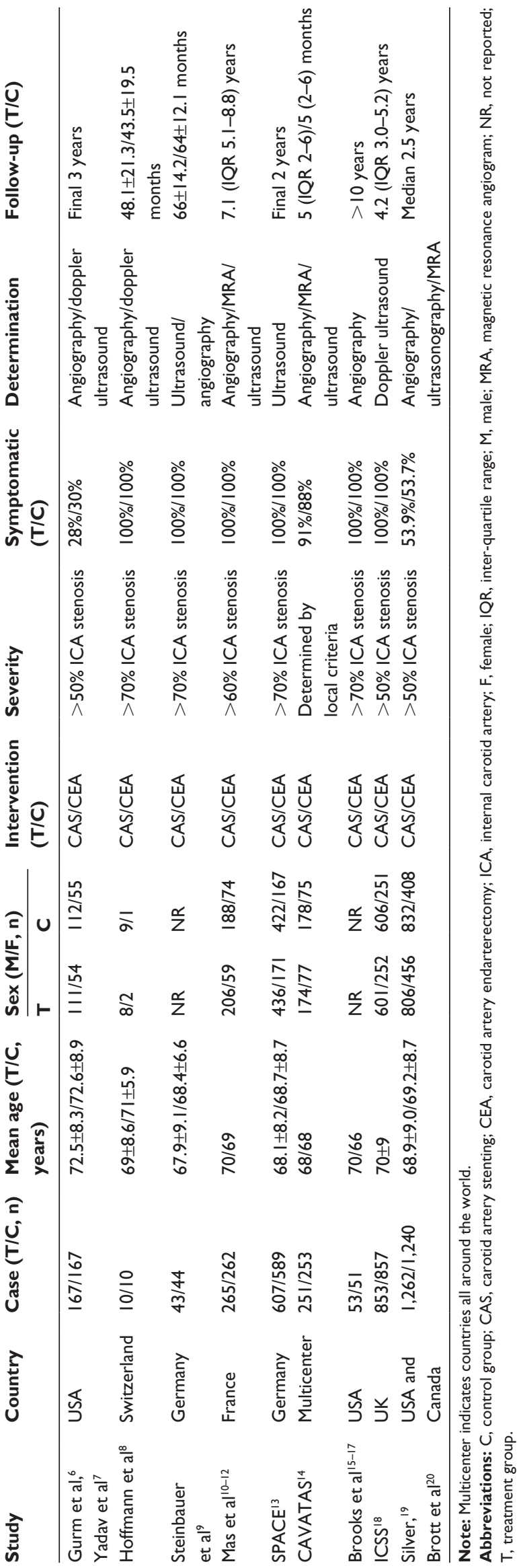




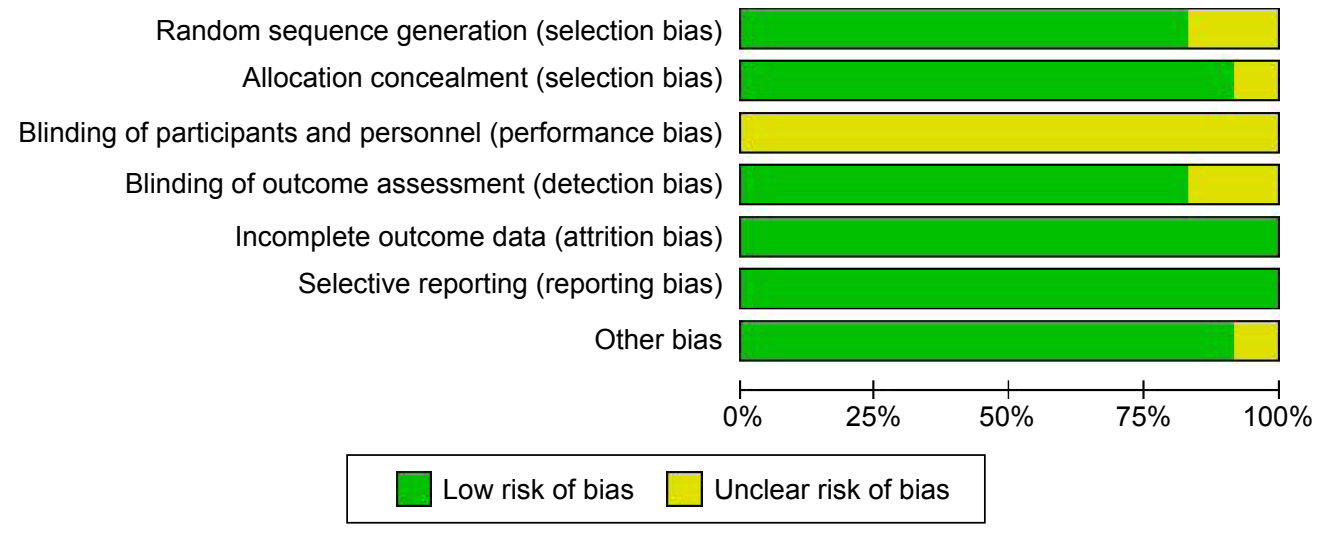

Figure 2 Summary of methodological quality assessment results.

in fixed-effects model showed that there was no significant difference between CAS and CEA during periprocedural 30 days $\left(I^{2}=0 \%, \mathrm{RR}=1.22,95 \% \mathrm{CI}: 0.69-2.14, P=0.50\right)$, postprocedural 24 months $\left(I^{2}=0 \%, \mathrm{RR}=0.99,95 \% \mathrm{CI}\right.$ : 0.71-1.37, $P=0.93), 48$ months $\left(I^{2}=0 \%, \mathrm{RR}=1.07,95 \% \mathrm{CI}\right.$ : $0.89-1.29, P=0.48)$, and $>48$ months $\left(I^{2}=0 \%, \mathrm{RR}=1.23,95 \%\right.$ CI: $1.00-1.52, P=0.05)$, as shown in Figure 3.

\section{Stroke}

According to the different follow-up period, a subgroup analysis including periprocedural 30 days, postprocedural 24,48 , and $>48$ months was conducted. Meta-analysis results in fixed-effects model showed that CAS was associated with a higher stroke incidence during periprocedural 30 days $\left(I^{2}=37 \%, \mathrm{RR}=1.62,95 \% \mathrm{CI}: 1.31-2.00, P<0.0001\right)$, 48 months $\left(I^{2}=0 \%, \mathrm{RR}=1.37,95 \%\right.$ CI: $\left.1.11-1.70, P=0.003\right)$, and $>48$ months $\left(I^{2}=20 \%, \mathrm{RR}=1.76,95 \% \mathrm{CI}: 1.34-2.31\right.$, $P<0.0001)$ than CEA, whereas there was no significant difference between the groups during postprocedural 24 months $\left(I^{2}=0 \%, \mathrm{RR}=1.08,95 \% \mathrm{CI}: 0.80-1.47, P=0.60\right)$, as shown in Figure 4.

Subgroup analysis of the long-term effects also included periprocedural stroke incidence. To avoid a repeated analysis of periprocedural stroke incidence in postprocedural 24,48 , and $>48$ months, another subgroup analysis excluding periprocedural incidence was also conducted. It revealed that there was no significant difference between CAS and CEA during periprocedural 30 days to postprocedural $24\left(I^{2}=0 \%, \mathrm{RR}=0.98,95 \% \mathrm{CI}: 0.60-1.60\right.$, $P=0.94)$ months and 48 months $\left(I^{2}=0 \%, \mathrm{RR}=1.07,95 \%\right.$ CI: $0.78-1.47, P=0.67)$. While, during periprocedural 30 days to postprocedural $>48$ months, the difference was statistically significant $\left(I^{2}=44 \%, \mathrm{RR}=1.58,95 \% \mathrm{CI}\right.$ : $1.11-2.23, P=0.01)$.

\section{Myocardial infarction}

Subgroup analysis including periprocedural 30 days, postprocedural 12 and 36 months was performed. Metaanalysis results in fixed-effects model showed that compared with CEA, CAS achieved a decreased incidence of myocardial infarction (MI) during periprocedural 30 days $\left(I^{2}=0 \%, \mathrm{RR}=0.44,95 \% \mathrm{CI}: 0.26-0.75, P=0.003\right)$, whereas the difference between the groups did not reach statistical significance during postprocedural 12 months $\left(I^{2}=0 \%, \mathrm{RR}=0.41,95 \% \mathrm{CI}: 0.15-1.08, P=0.07\right)$ and 36 months ( $\mathrm{RR}=0.64,95 \% \mathrm{CI}: 0.29-1.44, P=0.28)$, as shown in Figure 5.

\section{Disabling stroke and death}

Subgroup analysis including periprocedural 30 days, postprocedural 24 , and $>24$ months was performed. Metaanalysis results in the fixed-effects model showed that there was no significant difference between the groups during periprocedural 30 days $\left(I^{2}=0 \%, \mathrm{RR}=1.19,95 \% \mathrm{CI}\right.$ : $0.85-1.67, P=0.32)$, postprocedural 24 months $\left(I^{2}=50 \%\right.$, $\mathrm{RR}=1.30,95 \% \mathrm{CI}: 0.93-1.82, P=0.13)$, and $>24$ months $\left(I^{2}=48 \%, \mathrm{RR}=1.02,95 \% \mathrm{CI}: 0.84-1.22, P=0.87\right)$, as shown in Figure 6.

\section{Other major complications}

Compared with CEA, CAS was associated with a significant decrease in periprocedural cranial nerve palsy $\left(I^{2}=0 \%\right.$, $\mathrm{RR}=0.09,95 \%$ CI: $0.04,0.22, P<0.00001)$ and hematoma $\left(I^{2}=41 \%, \mathrm{RR}=0.31,95 \% \mathrm{CI}: 0.14,0.68, P=0.003\right)$, whereas it was associated with a significant increase in bradycardia or hypotension $\left(I^{2}=0 \%, \mathrm{RR}=8.45,95 \% \mathrm{CI}: 2.91-24.58\right.$, $P<0.0001)$. Besides, there was no significant difference in aspects of transient ischemic attack $\left(I^{2}=11 \%, \mathrm{RR}=1.58\right.$, 95\% CI: $0.93-2.68, P=0.09)$, restenosis $\left(I^{2}=0 \%, \mathrm{RR}=2.22\right.$, 


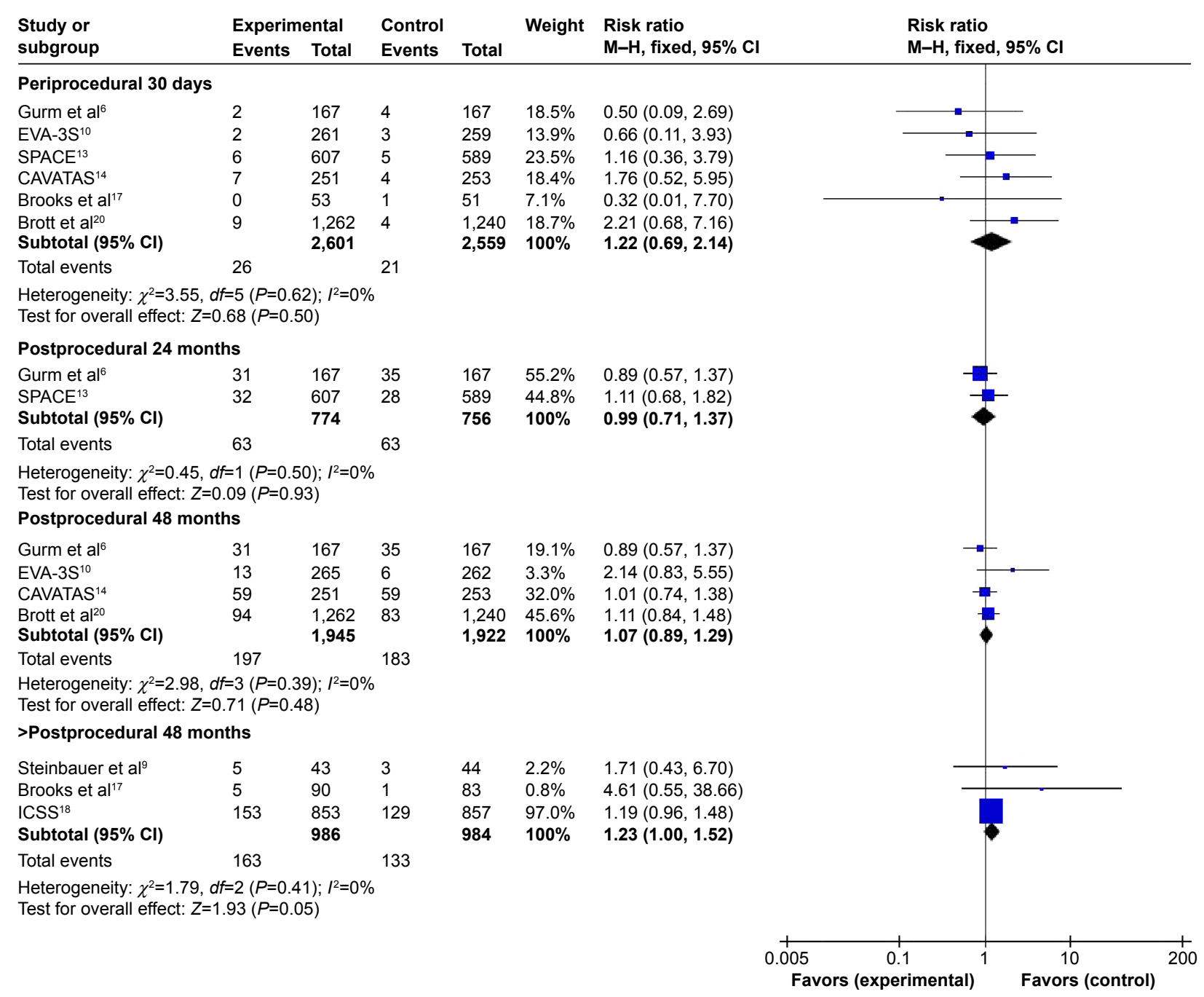

Figure 3 Meta-analysis of periprocedural and postprocedural death.

Abbreviations: $\mathrm{M}-\mathrm{H}$, Mantel-Haenszel; $\mathrm{Cl}$, confidence interval.

95\% CI: $0.51-9.60, P=0.29)$, arterial occlusion or thrombosis $\left(I^{2}=23 \%, \mathrm{RR}=1.49,95 \% \mathrm{CI}: 0.42-5.27, P=0.54\right)$, and infection $\left(I^{2}=0 \%, \mathrm{RR}=0.60,95 \% \mathrm{CI}: 0.08,4.54, P=0.62\right)$, as shown in Figure 7.

\section{Hospital stay}

Three trials ${ }^{7,8,17}$ reported the data of hospital stay, and the meta-analysis in random-effects model showed that there was no significant difference between CAS and CEA $\left(I^{2}=62 \%\right.$, mean difference $=-2.08,95 \% \mathrm{CI}:-4.47$ to -0.32 , $P=0.09)$.

\section{Publication bias}

Inverted funnel plots indicated that low risks of publication bias existed in the outcomes of death, stroke, and other major complications (Figure 8).

\section{Discussion}

The critical meta-analysis including 16 articles of nine RCTs with follow-up periods ranged from procedural 30 days to postprocedural 10 years. The pooled analysis altered that CAS was associated with increased risks of stroke compared with CEA during periprocedural 30 days and after postprocedural 4 years. And it confirmed the findings that higher risks of nondisabling stroke and bradycardia or hypotension, and a lower risk of MI were associated with CAS than CEA in the periprocedural period.

The estimated stroke rates were $6.19 \%, 9.79 \%, 9.56 \%$, and $12.89 \%$, respectively, at postprocedural 30 days, 2 years, 4 years and $>4$ years in the CAS group, compared with $3.82 \%, 9 \%, 6.97 \%$, and $7.33 \%$ in the CEA group. Due to the loss to follow-up and the reduction of available cases, total stroke rate of both was increasing during follow-up period, 


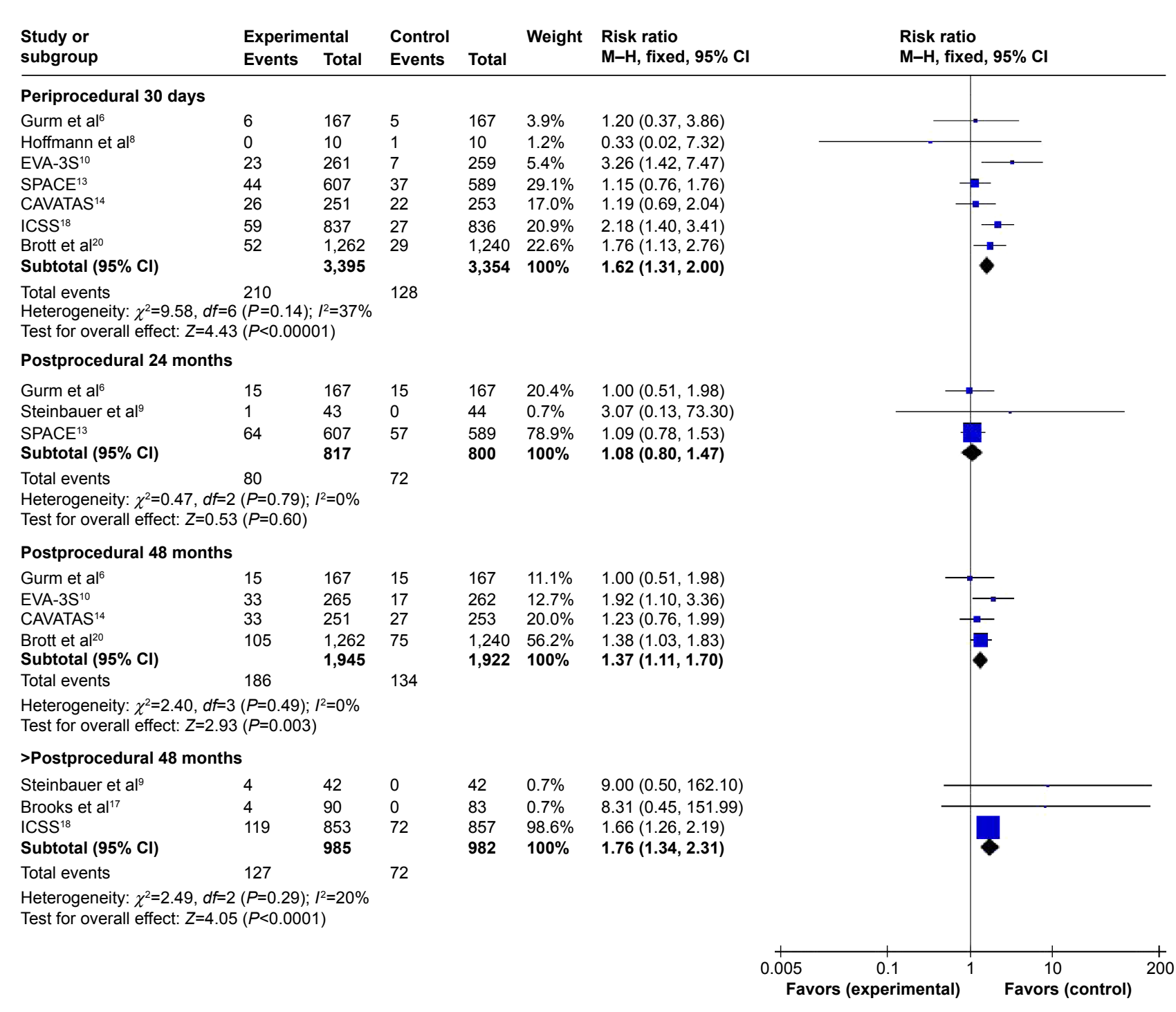

Figure 4 Meta-analysis of periprocedural and postprocedural stroke.

Abbreviations: $\mathrm{M}-\mathrm{H}$, Mantel-Haenszel; $\mathrm{Cl}$, confidence interval.

while CAS was always having a higher rate than CEA. In order to investigate the long-term effect of them, we further conducted a subgroup analysis including isolated data during procedural 30 days to final follow-up. The results revealed that CAS had a higher rate of stroke after postprocedural 4 years, based on the fact that there was no significant difference in outcomes of death and disabling stroke all the time. So it was clear that CAS had higher risks of nondisabling stroke during short-term of periprocedural 30 days, long-term of $>$ postprocedural 4 years, and overall period of follow-up, while a comparable risk during mid-term with CEA.

And CAS was demonstrated to achieve less cranial nerve palsy than CEA. However, it is hard for the patients in CEA group to identify a cranial nerve palsy from a stroke, so the actual stroke rate in CAS group might be even higher. Although the underlying mechanism was unclear, several studies reported that the increased incidence of stroke was mainly occurring in the contralateral carotid or vertebrobasilar territory. ${ }^{14,25}$ As is known, carotid artery and vertebrobasilar artery anastomoses each other through the circle of Willis (coW), and both the structure and function of coW are very important for blood supply of the whole brain. On the whole, local carotid artery surgeries may have different effects on the function of coW, ${ }^{26}$ and these different influences might be the major causes of therapeutic differences. A study including 139 patients reported that after carotid revascularization, the average diameter of ipsilateral precommunicating anterior cerebral artery (A1) increased $0.1 \mathrm{~mm}$, while ipsilateral and contralateral posterior communicating artery ( $\mathrm{PCoA})$ decreased $0.12 \mathrm{~mm}$ and $0.08 \mathrm{~mm} .{ }^{25}$ But, CAS led to much more diameter changes than CEA, with a maximum increase of $0.16 \mathrm{~mm}$ and decrease of $0.09 \mathrm{~mm}$. After revascularization 


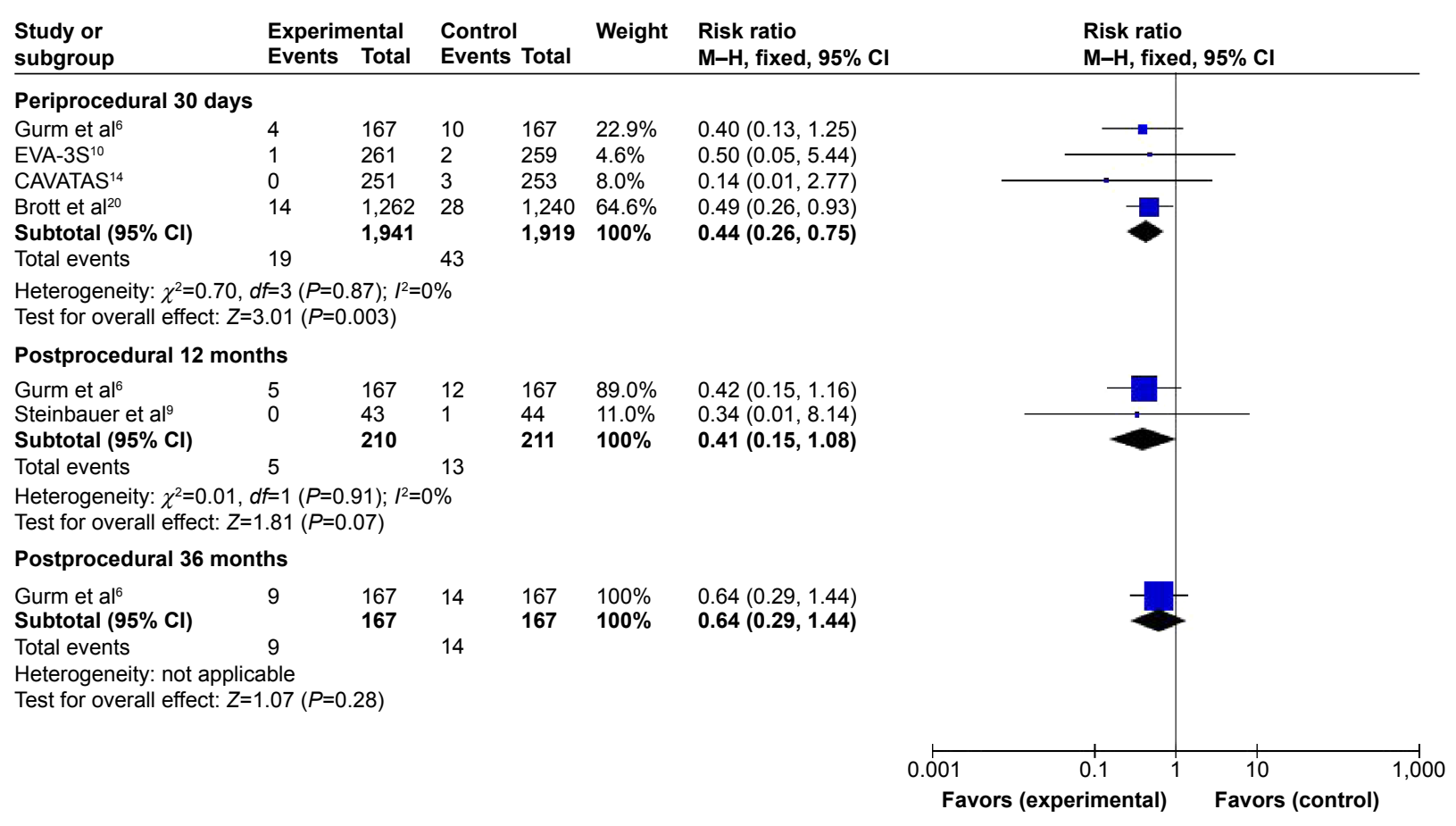

Figure 5 Meta-analysis of periprocedural and postprocedural myocardial infarction. Abbreviations: $\mathrm{M}-\mathrm{H}$, Mantel-Haenszel; $\mathrm{Cl}$, confidence interval.

stenosis in carotid artery was eliminated, A1 perfusion was increased, and PCoA perfusion was back to normal. Although they were relatively small changes, they had marked hemodynamic effects. ${ }^{27}$
Still we cannot rule out the conclusion that the much more changes caused by CAS increased the higher risk of stroke than CEA because of the insufficient data and limitations from the study. ${ }^{25}$ While the other study demonstrated that the coW is

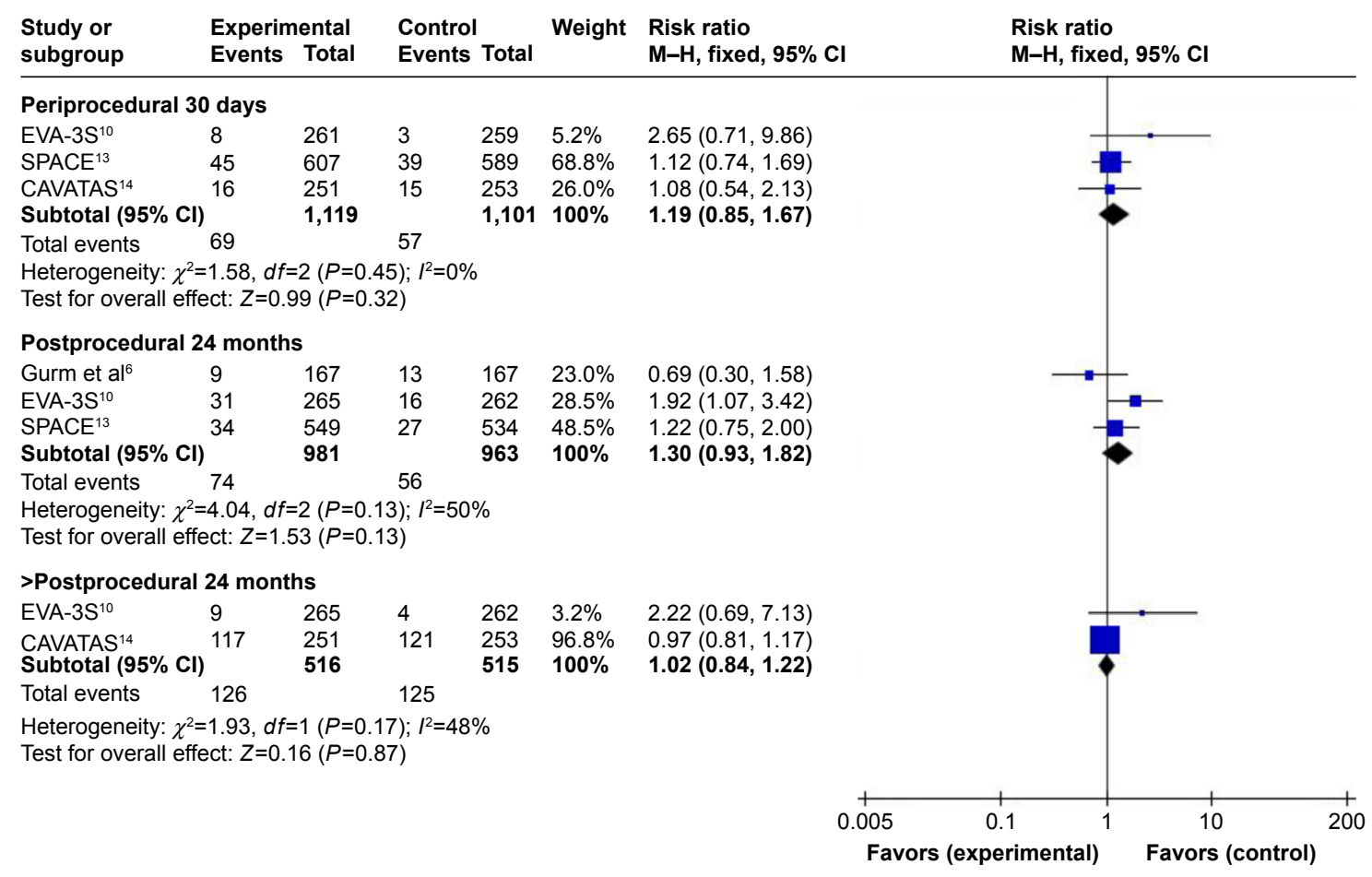

Figure 6 Meta-analysis of periprocedural disabling stroke and death. Abbreviations: $\mathrm{M}-\mathrm{H}$, Mantel-Haenszel; $\mathrm{Cl}$, confidence interval. 


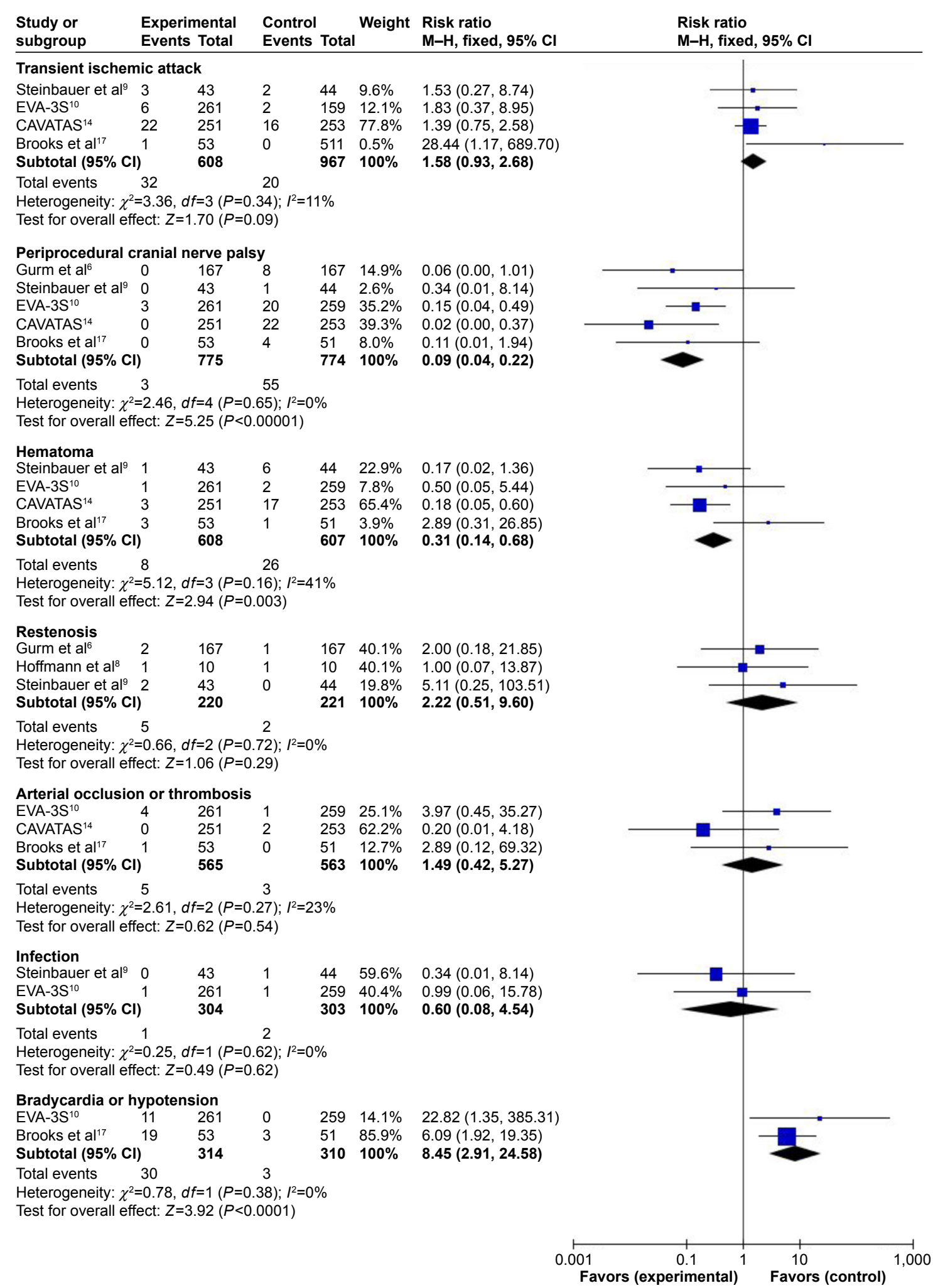

Figure 7 Meta-analysis of other periprocedural complications.

Abbreviations: $\mathrm{M}-\mathrm{H}$, Mantel-Haenszel; $\mathrm{Cl}$, confidence interval.

plastic, ${ }^{28}$ except for inborn variation, stenting would alter the flow pattern, and nearly one-third of the subjects adopted CAS had a blockade of A1, PCoA or precommunicating posterior cerebral artery (P1) at postprocedural 1 week..$^{29}$ Meanwhile, our meta-analysis mainly included patients of $>50 \%$ ipsilateral stenosis, and most of them were symptomatic, who had high possibilities of variant structure and impaired function of coW compared with asymptomatic patients, although detailed 
A

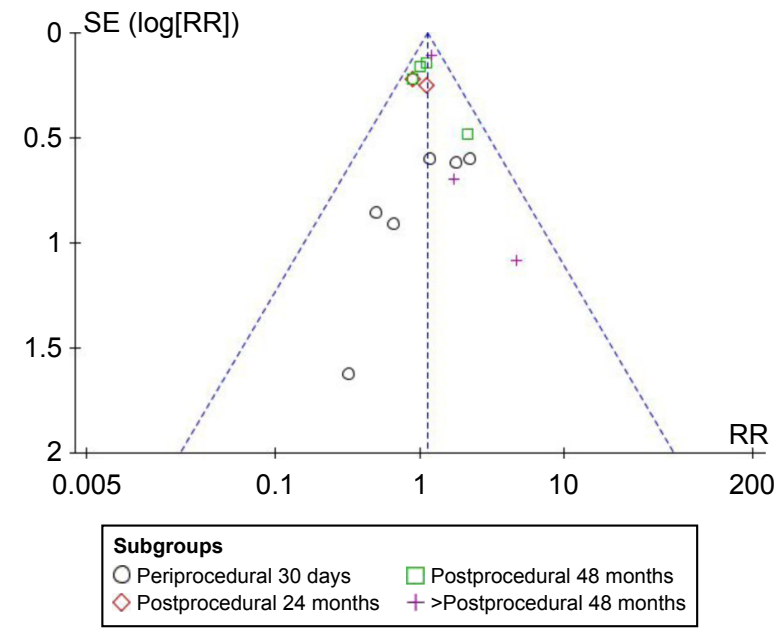

B

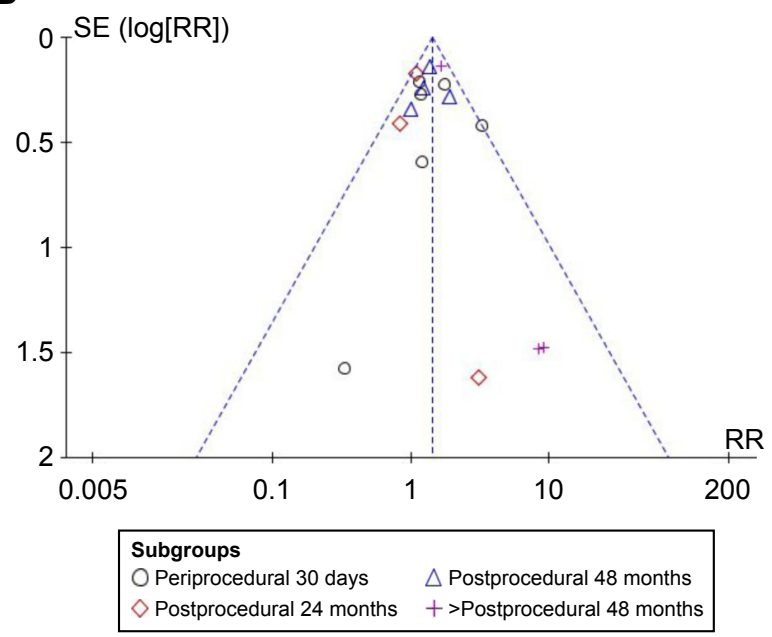

Figure $\mathbf{8}$ Inverted funnel plots indicating low risks of publication bias. Notes: (A) Death; (B) stroke.

Abbreviations: RR, risk ratio; SE, standard error.

information is absent. Therefore, hemodynamic instability such as bradycardia or hypotension together with significantly altered coW flow pattern may to some extent explain the difference risk of nondisabling stroke between CAS and CEA.

Our meta-analysis also revealed that CAS had a significant high risk of stroke than CEA on long-term effects. In the current analysis, patients had an average age more than 67 years, and studies demonstrated that age was an independent predictor of stroke in CAS other than CEA. ${ }^{18,30,31}$ Patients $>70$ years old had a significantly increased risk of CAS in aspects of procedural stroke or death or ipsilateral stroke. After 48 months, patients including in the metaanalysis had an average age of nearly $71-72$ years. So taking everything together, we may conclude that no matter to adopt or already adopted CAS, patients aged $>70$ years would suffer higher risk than CEA.

For other major complications, CAS significantly reduced the incidence of local hematoma. There was no significant difference in the aspects of infection, artery occlusive thrombosis, infection, restenosis, transient ischemic attack, and hospital stay. So as a minimally invasive surgery, CAS did not have obvious advantages than CEA, except for a decreased incision size, while clinically it seemed to be difficult for surgeons to perform a reoperation in recurrent patients who had undergone CAS.

Although the overall quality of the included RCTs was good, some other limitations existed: 1) stent material and type were not reported in detail, and different stent might have its special characters; ${ }^{32}$ ) an accurate diagnosis of MI should be based on symptom, electrocardiogram, and Q-wave situation. However, most of the studies did not report a standard diagnosis method, and this might lead to potential bias; 3) surgeon's experience may to affect the therapeutic effects. While, it was still without confirmed conclusions, ${ }^{33} 4$ ) most of the patients were symptomatic, so the results and conclusions were mainly based on data of symptomatic patients. Their comparative efficacy on asymptomatic patients was inconclusive; 5) Stenosis location and coW structure may be the most important factors to influence future clinical judgment and choice, whereas currently RCTs did not involve them; and 6) only on one study performed cost analysis, while it only compared medical cost and did not add the cost of stent. ${ }^{15}$ Actually, CAS had a higher total cost than CEA.

\section{Conclusion}

CAS reduced hematoma, periprocedural MI, and cranial nerve palsy, while it was associated with a higher risk of nondisabling stroke of both short-term and long-term period in elderly patients with severe and symptomatic carotid stenosis. After considering age and survival time, we suggest that the choice on CAS or CEA in symptomatic patients should take into account coW situation, financial condition, and cosmetic requirement.

\section{Disclosure}

The authors report no conflicts of interest in this work.

\section{References}

1. Go AS, Mozaffarian D, Roger VL, et al. Heart disease and stroke statistics-2014 update: a report from the American Heart Association. Circulation. 2014;129(3):e28.

2. Schneider AT, Kissela B, Woo D, et al. Ischemic stroke subtypes: a population-based study of incidence rates among blacks and whites Stroke. 2004;35(7):1552-1556. 
3. Guay J. Endovascular stenting or carotid endarterectomy for treatment of carotid stenosis: a meta-analysis. J Cardiothorac Vasc Anesth. 2011;25(6):1024-1029.

4. Chaturvedi S, Bruno A, Feasby T, et al. Carotid endarterectomy - an evidence-based review: report of the Therapeutics and Technology Assessment Subcommittee of the American Academy of Neurology[J]. Am J Ophthalmol. 2006;141(1):238-239.

5. European Carotid Surgery Trialists' Collaborative Group. Randomised trial of endarterectomy for recently symptomatic carotid stenosis: final results of the MRC European Carotid Surgery Trial (ECST)[J]. Lancet. 1998;351(9113):1379-1387.

6. Gurm HS, Yadav JS, Fayad P, et al. Long-term results of carotid stenting versus endarterectomy in high-risk patients[J]. $N$ Engl J Med. 2008;358(15):1572-1579.

7. Yadav JS, Wholey MH, Kuntz RE, et al. Protected carotid-artery stenting versus endarterectomy in high-risk patients[J]. N Engl J Med. 2004;351(15):1493-1501

8. Hoffmann A, Engelter S, Taschner C, et al. Carotid artery stenting versus carotid endarterectomy-a prospective randomised controlled single-centre trial with long-term follow-up (BACASS)[J]. Neurol Psychiatr. 2008;159:84-89.

9. Steinbauer MGM, Pfister K, Greindl M, et al. Alert for increased longterm follow-up after carotid artery stenting: results of a prospective, randomized, single-center trial of carotid artery stenting vs carotid endarterectomy[J]. J Vasc Surg. 2008;48(1):93-98.

10. Mas JL, Trinquart L, Leys D, et al. Endarterectomy Versus Angioplasty in Patients with Symptomatic Severe Carotid Stenosis (EVA-3S) trial: results up to 4 years from a randomised, multicentre trial[J]. Lancet Neurol. 2008;7(10):885-892.

11. Mas JL, Arquizan C, Calvet D, et al. Long-term follow-up study of endarterectomy versus angioplasty in patients with symptomatic severe carotid stenosis trial[J]. Stroke. 2014;45(9):2750-2756.

12. Mas JL, Chatellier G, Beyssen B, et al. Endarterectomy versus stenting in patients with symptomatic severe carotid stenosis[J]. N Engl J Med. 2006;355(16):1660-1671.

13. Eckstein HH, Ringleb P, Allenberg JR, et al. Results of the StentProtected Angioplasty versus Carotid Endarterectomy (SPACE) study to treat symptomatic stenoses at 2 years: a multinational, prospective, randomised trial[J]. Lancet Neurol. 2008;7(10): 893-902.

14. Ederle J, Bonati LH, Dobson J, et al. Endovascular treatment with angioplasty or stenting versus endarterectomy in patients with carotid artery stenosis in the Carotid and Vertebral Artery Transluminal Angioplasty Study (CAVATAS): long-term follow-up of a randomised trial[J]. Lancet Neurol. 2009;8(10):898-907.

15. Brooks WH, McClure RR, Jones MR, Coleman TC, Breathitt L. Carotid angioplasty and stenting versus carotid endarterectomy: randomized trial in a community hospital[J]. J Am Coll Cardiol. 2001;38(6): 1589-1595(7).

16. Brooks WH, Mcclure RR, Jones MR, Coleman TL, Breathitt L. Carotid angioplasty and stenting versus carotid endarterectomy for treatment of asymptomatic carotid stenosis: a randomized trial in a community hospital[J]. Neurosurgery. 2004;54(2):318-325.
17. Brooks WH, Jones MR, Gisler P, et al. Carotid Angioplasty With Stenting Versus Endarterectomy : 10-Year Randomized Trial in a Community Hospital[J]. JACC Cardiovasc Interv. 2014;7(2):163-168.

18. Bonati LH, Dobson J, Featherstone RL, et al. Long-term outcomes after stenting versus endarterectomy for treatment of symptomatic carotid stenosis: the International Carotid Stenting Study (ICSS) randomised trial.[J]. Lancet. 2015;385(14):529-538.

19. Silver FL. Safety of stenting and endarterectomy by symptomatic status in the Carotid Revascularization Endarterectomy Versus Stenting Trial (CREST).[J]. Stroke. 2011;54(3):280.

20. Brott TG, Hobson RW, Howard G, et al. Stenting versus endarterectomy for treatment of carotid-artery stenosis[J]. N Engl J Med. 2010;363(1):11-23.

21. Murad MH, Shahrour A, Shah ND, Montori VM, Ricotta JJ. A systematic review and meta-analysis of randomized trials of carotid endarterectomy vs stenting[J]. J Vasc Surg. 2011;53(3):792-797.

22. Bonati LH, Lyrer P, Ederle J, et al. Percutaneous transluminal balloon angioplasty and stenting for carotid artery stenosis[J]. Cochrane Database Syst Rev. 2012;9:CD000515.

23. Higgins JPT, Green S. Cochrane Handbook for Systematic Reviews of Interventions, 5.1. 0 (updated March 2011). The Cochrane Collaboration 2011[J]. Available from: www.cochrane-handbook.org; 2011.

24. Moher D, Shamseer L, Clarke M, et al. Preferred reporting items for systematic review and meta-analysis protocols (PRISMA-P) 2015 statement[J]. Syst Rev. 2015;4(1):1.

25. Bost RBC, Hendrikse J, Algra A, et al. Effects of carotid endarterectomy or stenting on arterial diameters in the circle of Willis[J]. J Stroke Cerebrovasc Dis. 2014;23(4):699-705.

26. Cassot F, Vergeur V, Bossuet P, Hillen B, Zagzoule M, Marc-Vergnes JP. Effects of Anterior Communicating Artery Diameter on Cerebral Hemodynamics in Internal Carotid Artery Disease A Model Study[J]. Circulation. 1995;92(10):3122-3131.

27. Cao Q, Zhang J, Xu G. Hemodynamic Changes and Baroreflex Sensitivity Associated with Carotid Endarterectomy and Carotid Artery Stenting[J]. Interv Neurol. 2014;3(1):13-21.

28. Chuang YM, Guo W, Lin CP. Appraising the plasticity of the circle of Willis: a model of hemodynamic modulation in cerebral arteriovenous malformations[J]. Eur Neurol. 2010;63(5):295-301.

29. Chuang YM, Lin CP, Wong HF, et al. Plasticity of circle of Willis: a longitudinal observation of flow patterns in the circle of Willis one week after stenting for severe internal carotid artery stenosis[J]. Cerebrovasc Dis. 2009;27(6):572-578.

30. Economopoulos KP, Sergentanis TN, Tsivgoulis G, Mariolis AD, Stefanadis C. Carotid artery stenting versus carotid endarterectomy a comprehensive meta-analysis of short-term and long-term outcomes[J]. Stroke. 2011;42(3):687-692.

31. Bonati L. Stenting or Endarterectomy for patients with symptomatic carotid stenosis[J]. Neurol Clin. 2015;33(2):459-474.

32. He D, Liu W, Zhang T. The Development of Carotid Stent Material[J]. Interv Neurol. 2014;3(2):67-77.

33. Bangalore S, Kumar S, Wetterslev J, et al. Carotid artery stenting vs carotid endarterectomy: meta-analysis and diversity-adjusted trial sequential analysis of randomized trials[J]. Arch Neurol. 2011;68(2):172-184.
Clinical Interventions in Aging

\section{Publish your work in this journal}

Clinical Interventions in Aging is an international, peer-reviewed journal focusing on evidence-based reports on the value or lack thereof of treatments intended to prevent or delay the onset of maladaptive correlates of aging in human beings. This journal is indexed on PubMed Central, MedLine,

\section{Dovepress}

CAS, Scopus and the Elsevier Bibliographic databases. The manuscript management system is completely online and includes a very quick and fair peer-review system, which is all easy to use. Visit http://www.dovepress. com/testimonials.php to read real quotes from published authors. 